Graphs and Combinatorics (2008) 24:291-301

Digital Object Identifier (DOI) 10.1007/s00373-008-0795-7

\title{
Hadwiger Number and the Cartesian Product of Graphs
}

\author{
L. Sunil Chandran ${ }^{1}$, Alexandr Kostochka ${ }^{2,3}$, J. Krishnam Raju ${ }^{4}$ \\ 1 Department of Computer Science and Automation, Indian Institute of Science, Bangalore \\ 560012. e-mail: sunil@csa.iisc.ernet.in,sunil.cl@gmail.com \\ 2 University of Illinois at Urbana-Champaign, IL 61801, USA. \\ 3 Institute of Mathematics, Novosibirsk 630090, Russia. e-mail: kostochk@math.uiuc.edu \\ 4 David R. Cheriton School of Computer Science, University of Waterloo. \\ e-mail:krjampan@cs.uwaterloo.ca
}

\begin{abstract}
The Hadwiger number $\eta(G)$ of a graph $G$ is the largest integer $n$ for which the complete graph $K_{n}$ on $n$ vertices is a minor of $G$. Hadwiger conjectured that for every graph $G, \eta(G) \geq \chi(G)$, where $\chi(G)$ is the chromatic number of $G$. In this paper, we study the Hadwiger number of the Cartesian product $G \square H$ of graphs.

As the main result of this paper, we prove that $\eta\left(G_{1} \square G_{2}\right) \geq h \sqrt{l}(1-o(1))$ for any two graphs $G_{1}$ and $G_{2}$ with $\eta\left(G_{1}\right)=h$ and $\eta\left(G_{2}\right)=l$. We show that the above lower bound is asymptotically best possible when $h \geq l$. This asymptotically settles a question of $Z$. Miller (1978).

As consequences of our main result, we show the following:

1. Let $G$ be a connected graph. Let $G=G_{1} \square G_{2} \square \ldots \square G_{k}$ be the (unique) prime factorization of $G$. Then $G$ satisfies Hadwiger's conjecture if $k \geq 2 \log \log \chi(G)+c^{\prime}$, where $c^{\prime}$ is a constant. This improves the $2 \log \chi(G)+3$ bound in [2].

2. Let $G_{1}$ and $G_{2}$ be two graphs such that $\chi\left(G_{1}\right) \geq \chi\left(G_{2}\right) \geq c \log ^{1.5}\left(\chi\left(G_{1}\right)\right)$, where $c$ is a constant. Then $G_{1} \square G_{2}$ satisfies Hadwiger's conjecture.

3. Hadwiger's conjecture is true for $G^{d}$ (Cartesian product of $G$ taken $d$ times) for every graph $G$ and every $d \geq 2$. This settles a question by Chandran and Sivadasan [2]. (They had shown that the Hadiwger's conjecture is true for $G^{d}$ if $d \geq 3$ ).
\end{abstract}

Key words. Hadwiger Number, Hadwiger's Conjecture, Graph Cartesian product, Minor, Chromatic number.

\section{Introduction}

\subsection{General Definitions and Notation}

In this paper we only consider undirected simple graphs, i.e., graphs without multiple edges and loops. For a graph $G$, we use $V(G)$ to denote its vertex set and $E(G)$ to denote its edge set.

* Research of this author is supported in part by NSF grant DMS-0650784 and grant 0601-00694 of the Russian Foundation for Basic Research. 
A $k$-coloring of a graph $G(V, E)$ is a function $f: V \rightarrow\{1,2, \ldots, k\}$. A $k$ coloring $f$ is proper if for all edges $(x, y)$ in $G, f(x) \neq f(y)$. A graph is $k$-colorable if it has a proper $k$-coloring. The chromatic number $\chi(G)$ is the least $k$ such that $G$ is $k$-colorable.

Let $S_{1}$ and $S_{2}$ be non-empty disjoint subsets of $V(G)$. We say that $S_{1}$ and $S_{2}$ are adjacent in $G$ if there exists an edge $(u, v) \in E(G)$ such that $u \in S_{1}$ and $v \in S_{2}$. The edge $(u, v)$ is said to connect $S_{1}$ and $S_{2}$.

Contraction of an edge $e=(x, y)$ is the replacement of the vertices $x$ and $y$ with a new vertex $z$, whose incident edges are the edges other than $e$ that were incident to $x$ or $y$. The resulting graph denoted by G.e may be a multigraph, but since we are only interested in simple graphs, we discard any parallel edges.

A minor $M$ of $G(V, E)$ is a graph obtained from $G$ by a sequence of contractions of edges and deletions of edges and vertices. We call $M$ a minor of $G$ and write $M \preceq G$.

It is not difficult to verify that $M$ is a minor of $G$ if and only if for each vertex $x \in V(M)$, there exists a set $V_{x} \subseteq V(G)$ such that (1) every $V_{x}$ induces a connected subgraph of $G$, (2) all $V_{x}$ are disjoint, and (3) for each $(x, y) \in E(M), V_{x}$ is adjacent to $V_{y}$ in $G$.

The Hadwiger number $\eta(G)$ is the largest integer $h$ such that the complete graph on $h$ vertices $K_{h}$ is a minor of $G$. Since every graph on at most $h$ vertices is a minor of $K_{h}$, it is easy to see that $\eta(G)$ is the largest integer such that any graph on at most $\eta(G)$ vertices is a minor of $G$. Hadwiger [6] conjectured that for every graph $G, \eta(G) \geq \chi(G)$, where $\chi(G)$ is the chromatic number of $G$.

In other words, Hadwiger's conjecture states that if $\eta(G) \leq k$, then $G$ is $k$-colorable. It is known to hold for small $k$. Graphs of Hadwiger number at most 2 are the forests. By a theorem of Dirac [5], the graphs with Hadwiger number at most 3 are the series-parallel graphs. Graphs with Hadwiger number at most 4 are characterized by Wagner [19]. The case $k=4$ of the conjecture implies the Four Color Theorem because any planar graph has no $K_{5}$ minor. On the other hand, Hadwiger's conjecture for the case $k=4$ follows from the Four Color Theorem and a structure theorem of Wagner [19]. Hadwiger's conjecture for $k=5$ was settled by Robertson et al. [15]. The case $k=6$ onwards is still open.

Since Hadwiger's conjecture in the general case is still open, researchers have shown interest to derive lower bounds for Hadwiger number in terms of the chromatic number. Mader [13] showed (improving an earlier result of Wagner [20]) that for any graph $G, \eta(G) \geq \frac{\chi(G)}{16 \log (\chi(G))}$. Later, Kostochka [10] and Thomason [18] independently showed that there exists a constant $c$ such that for any graph $G$, $\eta(G) \geq \frac{\chi(G)}{c \cdot \sqrt{\log (\chi(G))}}$.

It is also known that Hadwiger's conjecture is true for almost all graphs on $n$ vertices.

Improving on previous results by other authors, Kühn and Osthus [12] showed that if the girth (i.e., the length of a shortest cycle) is at least $g$ for some odd $g$ and the minimum degree $\delta$ is at least 3 , then $\eta(G) \geq \frac{c(\delta)^{(g+1) / 4}}{\sqrt{\log \delta}}$. As a consequence of this result, Kühn and Osthus[12] showed that Hadwiger's conjecture is true for graphs of sufficiently large chromatic number with no 4-cycles. 


\subsection{The Cartesian Product of Graphs}

Let $G_{1}$ and $G_{2}$ be two undirected graphs, where the vertex set of $G_{1}$ is $\left\{0,1, \ldots, n_{1}-\right.$ $1\}$ and the vertex set of $G_{2}$ is $\left\{0,1, \ldots, n_{2}-1\right\}$. The Cartesian product, $G_{1} \square G_{2}$, of $G_{1}$ and $G_{2}$ is the graph with the vertex set $V=\left\{0,1, \ldots, n_{1}-1\right\} \times\left\{0,1, \ldots, n_{2}-1\right\}$ whose edge set is defined as follows. There is an edge between vertices $\langle i, j\rangle$ and $\left\langle i^{\prime}, j^{\prime}\right\rangle$ of $G_{1} \square G_{2}$ if and only if, either $j=j^{\prime}$ and $\left(i, i^{\prime}\right) \in E\left(G_{1}\right)$, or $i=i^{\prime}$ and $\left(j, j^{\prime}\right) \in E\left(G_{2}\right)$.

In other words, the Cartesian product can be viewed in the following way: let the vertices of $G_{1} \square G_{2}$ be partitioned into $n_{2}$ classes $W_{1}, \ldots, W_{n_{2}}$, where $W_{j}=$ $\left\{\langle 1, j\rangle, \ldots,\left\langle n_{1}, j\right\rangle\right\}$ induces a graph that is isomorphic to $G_{1}$, where the vertex $\langle i, j\rangle$ corresponds to vertex $i$ of $G_{1}$. If edge $\left(j, j^{\prime}\right)$ belongs to $G_{2}$ then the edges between classes $W_{j}$ and $W_{j^{\prime}}$ form a matching such that the corresponding vertices, i.e., $\langle i, j\rangle$ and $\left\langle i, j^{\prime}\right\rangle$, are matched. If edge $\left(j, j^{\prime}\right)$ is not present in $G_{2}$, then there is no edge between $W_{j}$ and $W_{j^{\prime}}$.

It is easy to verify that the Cartesian product is a commutative and associative operation on graphs. Due to the associativity, the product of graphs $G_{1}, \ldots, G_{k}$ can be simply written as $G_{1} \square \ldots \square G_{k}$ and has the following interpretation. If the vertex set of graph $G_{i}$ is $V_{i}=\left\{1, \ldots, n_{i}\right\}$, then $G_{1} \square \ldots \square G_{k}$ has the vertex set $V=$ $V_{1} \times V_{2} \times \ldots \times V_{k}$. There is an edge between vertex $\left\langle i_{1}, \ldots, i_{k}\right\rangle$ and vertex $\left\langle i_{1}^{\prime}, \ldots, i_{k}^{\prime}\right\rangle$ of $V$ if and only if there is a position $t, 1 \leq t \leq k$, such that $i_{1}=i_{1}^{\prime}, i_{2}=i_{2}^{\prime}, \ldots, i_{t-1}$ $=i_{t-1}^{\prime}, i_{t+1}=i_{t+1}^{\prime}, \ldots, i_{k}=i_{k}^{\prime}$, and the edge $\left(i_{t}, i_{t}^{\prime}\right)$ belongs to graph $G_{t}$.

We denote the product of graph $G$ taken $k$ times as $G^{k}$. It is easy to verify that if $G$ has $n$ vertices and $m$ edges, then $G^{k}$ has $n^{k}$ vertices and $m k \cdot n^{k-1}$ edges.

Well known examples of Cartesian products of graphs are the $d$-dimensional hypercube $Q_{d}$, which is isomorphic to $K_{2}^{d}$, and a $d$-dimensional grid, which is isomorphic to $P_{n}^{d}$, where $P_{n}$ is a simple path on $n$ vertices.

Unique Prime Factorization(UPF) of graphs: A graph $P$ is prime with respect to the Cartesian product if and only if $P$ has at least two vertices and it is not isomorphic to the product of two non-identity graphs, where an identity graph is the graph on a single vertex and having no edge. It is well-known that every connected undirected graph $G$ with at least two vertices has a UPF with respect to Cartesian product in the sense that if $G$ is not prime then it can be expressed in a unique way as a product of prime graphs ([7]). If $G$ can be expressed as the product $G_{1} \square G_{2} \square \ldots \square G_{k}$, where each $G_{i}$ is prime, then we say that the product dimension of $G$ is $k$. The UPF of a given connected graph $G$ can be found in $O(m \log (n))$ time, where $m$ and $n$ are the number of edges and number of vertices of $G$ respectively [1].

Imrich and Klavžar have published a book [7] dedicated exclusively to the study of graph products. Readers who are interested to get an introduction to the wealth of profound and beautiful results on graph products are referred to this book.

We will use the following result by Sabidussi [17] (which was rediscovered several times).

Lemma 1. $\chi\left(G_{1} \square G_{2}\right)=\max \left\{\chi\left(G_{1}\right), \chi\left(G_{2}\right)\right\}$. 


\subsection{Our Results}

The question of studying the Hadwiger number with respect to the Cartesian product was suggested by Miller in the open problems section of a 1978 paper [14]. He mentioned a couple of special cases (such as $\eta\left(C_{n} \square K_{2}\right)=4$ and $\eta\left(T \square K_{n}\right)=$ $n+1$, where $C_{n}$ and $T$ denote a cycle and a tree respectively) and left the general case open. In this paper, we answer this question asymptotically. We give the following results.

Result 1. Let $G_{1}$ and $G_{2}$ be two graphs with $\eta\left(G_{1}\right)=k_{1}$ and $\eta\left(G_{2}\right)=k_{2}$. Then $\eta\left(G_{1} \square G_{2}\right) \geq k_{1} \sqrt{k_{2}}(1-o(1))$. (Since the Cartesian product is commutative, we can assume that $k_{1} \geq k_{2}$ ). We demonstrate that this lower bound is asymptotically best possible.

We also show that in general, $\eta\left(G_{1} \square G_{2}\right)$ does not have any upper bound that depends only on $\eta\left(G_{1}\right)$ and $\eta\left(G_{2}\right)$ by demonstrating sequences of graphs $\left\{G_{1}(n)\right\}_{n=1}^{\infty}$ and $\left\{G_{2}(n)\right\}_{n=1}^{\infty}$ such that $\eta\left(G_{1}(n)\right)$ and $\eta\left(G_{2}(n)\right)$ are bounded, whereas $\eta\left(G_{1}(n) \square G_{2}(n)\right) \geq n$.

Remark. Note that if the average degrees of $G_{1}$ and $G_{2}$ are $d_{1}$ and $d_{2}$ respectively, then the average degree of $G_{1} \square G_{2}$ is $d_{1}+d_{2}$. In comparison, by Result 1 , the Hadwiger number of $G_{1} \square G_{2}$ grows much faster.

Hadwiger's conjecture for Cartesian products of graphs was studied in [2]. It was shown there that if the product dimension (number of factors in the unique prime factorization of $G$ ) is $k$, then Hadwiger's conjecture is true for $G$ if $k \geq 2 \log \chi(G)+3$. As a consequence of Result 1, we are able to improve this bound. We show the following.

Result 2. Let the (unique) prime factorization of $G$ be $G=G_{1} \square G_{2} \square \ldots \square G_{k}$. Then Hadwiger's conjecture is true for $G$ if $k \geq 2 \log (\log (\chi(G)))+c^{\prime}$, where $c^{\prime}$ is a constant.

Another consequence of Result 1 is that if $G_{1}$ and $G_{2}$ are two graphs such that $\chi\left(G_{2}\right)$ is not "too low" compared to $\chi\left(G_{1}\right)$, then Hadwiger's conjecture is true for $G_{1} \square G_{2}$. More precisely:

Result 3. If $\chi\left(G_{2}\right) \geq c \log ^{1.5}\left(\chi\left(G_{1}\right)\right)$, where $c$ is a constant, then Hadwiger's conjecture is true for $G_{1} \square G_{2}$.

It is easy to see that Result 3 implies the following: Let $G_{1}$ and $G_{2}$ be two graphs such that $\chi\left(G_{1}\right)=\chi\left(G_{2}\right)$. (For example, as in the case $G_{1}=G_{2}$ ). Then $G_{1} \square G_{2}$ satisfies Hadwiger's conjecture if $\chi\left(G_{1}\right)=\chi\left(G_{2}\right)=t$ is sufficiently large. ( $t$ has to be sufficiently large, because of the constant $c$ involved in Result 3 ). For this special case, namely $\chi\left(G_{1}\right)=\chi\left(G_{2}\right)$, we give a different proof (which does not depend on Result 1), to show that Hadwiger's conjecture is true for $G_{1} \square G_{2}$. This proof does not require that $\chi\left(G_{1}\right)$ be sufficiently large. 
Result 4. Let $G_{1}$ and $G_{2}$ be any graphs with $\chi\left(G_{1}\right)=\chi\left(G_{2}\right)$. Then Hadwiger's conjecture is true for $G_{1} \square G_{2}$.

It was shown in [2] that Hadwiger's conjecture is true for $G^{d}$, where $d \geq 3$, for any graph $G$. As a consequence of Result 4, we are able to sharpen this result.

Result 5. For any graph $G$ and every $d \geq 2$, Hadwiger's Conjecture is true for $G^{d}$.

Another author who studied the minors of the Cartesian product of graphs is Kotlov [11]. He showed that for every bipartite graph $G$, the strong product ([7]) $G \otimes K_{2}$ is a minor of $G \square C_{4}$. As a consequence of this he showed that $\eta\left(K_{2}^{d}\right) \geq 2^{\frac{d+1}{2}}$.

\section{Hadwiger Number for $\mathbf{G}_{1} \square \mathbf{G}_{2}$}

\subsection{Lower Bound on $\eta\left(G_{1} \square G_{2}\right)$}

The following Lemma is not difficult to prove.

Lemma 2. [2] If $M_{1} \preceq G_{1}$ and $M_{2} \preceq G_{2}$, then $M_{1} \square M_{2} \preceq G_{1} \square G_{2}$.

Let $G_{1}$ and $G_{2}$ be two graphs such that $\eta\left(G_{1}\right)=h$ and $\eta\left(G_{2}\right)=l$, with $h \geq l$. In this section we show that $\eta\left(G_{1} \square G_{2}\right) \geq h \sqrt{l}(1-o(1))$. Since by Lemma 2 , $K_{h} \square K_{l} \preceq G_{1} \square G_{2}$ it is sufficient to prove that $\eta\left(K_{h} \square K_{l}\right) \geq h \sqrt{l}(1-o(1))$.

Definition 1. An affine plane $\mathcal{A}$ of order $m$ is a family $\left\{A_{q, t}: q=1, \ldots, m+1, t=\right.$ $1, \ldots, m\}$ of $m$-elements subsets of an $m^{2}$-element set $A$ such that

$$
\left|A_{q, t} \cap A_{q^{\prime}, t^{\prime}}\right|=\left\{\begin{array}{l}
1 \text { if } q^{\prime} \neq q \\
0 \text { if } q^{\prime}=q \text { and } t^{\prime} \neq t .
\end{array}\right.
$$

The sets $A_{i t}$ are the lines of $\mathcal{A}$. By definition, for each $q$, the sets $A_{q, 1}, A_{q, 2}, \ldots$, $A_{q, m}$ are disjoint and form a partition of $A$. These sets are viewed as parallel lines.

The following fact is widely known (see, e.g., [16]):

Lemma 3. If $m$ is a prime power, then there exists an affine plane of order $m$.

It is also known that the set of prime numbers is quite dense. The following is a weakening of the result of Iwaniec and Pintz [9].

Lemma 4. For every sufficiently large positive $x$, the interval $\left[x, x+x^{0.6}\right]$ contains a prime number.

Corollary 1. For every sufficiently large positive $x$, the interval $\left[x-6 x^{0.9}, x\right]$ contains a number of the form $(p(p+1))^{2}$, where $p$ is some prime.

Theorem 1. $\eta\left(K_{h} \square K_{l}\right) \geq h \sqrt{l}(1-o(1))$. 


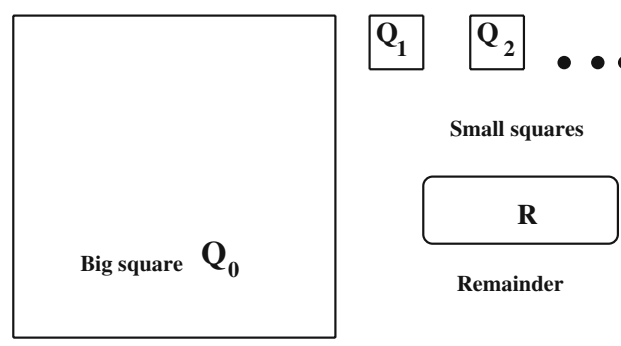

Fig. 1.

Proof. Consider $G=K_{h} \square K_{l}$, where $h \geq l$ and $l$ is large. Let $p$ be the maximum prime such that $l \geq(p(p+1))^{2}$. We view $K_{h} \square K_{l}$ as a set of $h$ copies of $K_{l}$. Suppose that $s(p-1)(2 p+1) / 2 \leq h<(s+1)(p-1)(2 p+1) / 2$. We neglect some $h-s(p-1)(2 p+1) / 2$ copies of $K_{l}$ and partition the remaining $s(p-1)(2 p+1) / 2$ copies into $s$ large groups of the same size, and each of these groups into $(p-1) / 2$ groups of size $2 p+1$. In other words, we consider $\mathcal{S}=\left\{K_{l}(i, j, m): i=1, \ldots, s, j=\right.$ $1, \ldots,(p-1) / 2, m=1, \ldots, 2 p+1\}$, where each $K_{l}(i, j, m)$ is a copy of $K_{l}$. For $i=1, \ldots, s$, let $\mathcal{S}_{i}=\left\{K_{l}(i, j, m): j=1, \ldots,(p-1) / 2 ; m=1, \ldots, 2 p+1\right\}$.

In $\mathcal{S}_{1}$, we will find $p^{2}(p-1)(2 p+1) / 2$ disjoint sets $M(1, j, m, t)(j=1, \ldots,(p-$ 1) $\left./ 2, m=1, \ldots, 2 p+1, t=1, \ldots, p^{2}\right)$ of size $(p+1)^{2}$. These sets will have the property that

(a) the subgraph $G(M(1, j, m, t))$ induced by $M(1, j, m, t)$ is connected;

(b) for any two quadruples $(1, j, m, t)$ and $\left(1, j^{\prime}, m^{\prime}, t^{\prime}\right)$, there is a vertex $v$ in $K_{l}$ such that each of $M(1, j, m, t)$ and $M\left(1, j^{\prime}, m^{\prime}, t^{\prime}\right)$ contains a copy of $v$ (in different copies of $K_{l}$, since our sets are disjoint).

If we manage this, then copying these sets for every $i=2, \ldots, s$, by (b), we will create $s p^{2}(p-1)(2 p+1) / 2=p^{2} h(1-o(1))$ disjoint sets $M(i, j, m, t)$ that satisfy

(a') the subgraph $G(M(i, j, m, t))$ induced by $M(i, j, m, t)$ is connected;

(b') for any two quadruples $(i, j, m, t)$ and $\left(i^{\prime}, j^{\prime}, m^{\prime}, t^{\prime}\right)$, there is a vertex $v$ in $K_{l}$ such that each of $M(i, j, m, t)$ and $M\left(i^{\prime}, j^{\prime}, m^{\prime}, t^{\prime}\right)$ contains a copy of $v$

So, we go after (a) and (b).

To achieve this, we view the set of vertices of each $K_{l}$ as the disjoint union of a "big" square $Q_{0}$ of size $p^{2} \times p^{2}$ with $2 p+1$ "small" squares $Q_{k}, k=1, \ldots, 2 p+1$ of size $p \times p$ and the remainder $R$ (of size $\left.l-p^{4}-(2 p+1) p^{2}=l-(p(p+1))^{2}\right)$ (see Fig. 1).

By Lemma 3, there exists an affine plane of order $p^{2}$. We consider the lines $A_{q, t}$ of this plane as subsets of the big square $Q_{0}$. For $q=1, \ldots,(p-1)(2 p+1) / 2$, we view the family $\left\{A_{q, 1}, A_{q, 2}, \ldots, A_{q, p^{2}}\right\}$ as a partition of the copy $Q_{0}(q)$ of the big square $Q_{0}$. If $q=(j-1)(2 p+1)+m$, then the set $A_{q, t}$ will be the main part of the future set $M(1, j, m, t)$. The whole line $A_{q, t}$ lies in one copy of $K_{l}$ and so $G\left(A_{q, t}\right)$ is connected. By the definition of the affine plane, if $q^{\prime}=\left(j^{\prime}-1\right)(2 p+1)+m^{\prime}$ and $q^{\prime} \neq q$, then the projections of the sets $A_{q, t}$ and $A_{q^{\prime}, t^{\prime}}$ on $Q_{0}$ intersect and for them (b) holds. Thus, we need only to take care of sets $M(1, j, m, t)$ and $M\left(1, j^{\prime}, m^{\prime}, t^{\prime}\right)$ when $j=j^{\prime}$ 
and $m=m^{\prime}$. So, our goal is to add $2 p+1$ vertices to each of $A_{(j-1)(2 p+1)+m, t}$ to provide (b) for $M(1, j, m, t)$ and $M\left(1, j, m, t^{\prime}\right)$.

Fix $j$ and $m$. For every $t=1, \ldots, p^{2}$, the set $M(1, j, m, t)$ will be obtained from $A_{(j-1)(2 p+1)+m, t}$ by adding a $(2 p+1)$-element subset of $\cup_{r=1}^{2 p+1} Q_{m}(j, r)$, where $Q_{m}(j, r)$ is the copy of $Q_{m}$ that is contained in $K_{l}(1, j, r)$. Every $t=1, \ldots, p^{2}$ can be written in the form $t=\left(a_{1}-1\right) p+a_{2}$, where $1 \leq a_{1}, a_{2} \leq p$. So, we include into $M(1, j, m, t)$ the entry $\left(a_{1}, a_{2}\right)$ of the square $Q_{m}(j, m)$. We call this vertex $F\left(a_{1}, a_{2}, j, m\right)$. Since $F\left(a_{1}, a_{2}, j, m\right)$ is in the same copy of $K_{l}$ as $A_{(j-1)(2 p+1)+m, t}$, it is adjacent to every vertex in this set. Let $C_{a}(j, r, m)$ and $R_{b}(j, r, m)$ denote the $a$ th column and the $b$ th row of the square $Q_{m}(j, r)$, respectively. If $t=\left(a_{1}-1\right) p+a_{2}$, then our set $M(1, j, m, t)$ will consist of $A_{(j-1)(2 p+1)+m, t}$, the vertex $F\left(a_{1}, a_{2}, j, m\right)$, the row $R_{a_{1}}\left(j, m+a_{2}, m\right)$ and the column $C_{a_{2}}\left(j, m-a_{1}, m\right)$, where the values $m+a_{2}$ and $m-a_{1}$ are calculated modulo $2 p+1$. Since $F\left(a_{1}, a_{2}, j, m\right)$ is adjacent to the $a_{2}$-s entry of the row $R_{a_{1}}\left(j, m+a_{2}, m\right)$ and to the $a_{1}$-s entry of the column $C_{a_{2}}\left(j, m-a_{1}, m\right)$, condition (a) holds. Since the projection on $Q_{m}$ of $R_{a_{1}}(j, m+$ $\left.a_{2}, m\right) \cup C_{a_{2}}\left(j, m-a_{1}, m\right)$ is a cross, (b) also holds.

This finishes the construction. It implies that the Hadwiger number of $G=$ $K_{h} \square K_{l}$, with $h \geq l$ is at least

$$
\left\lfloor\frac{h}{(p-1)(2 p+1) / 2}\right\rfloor p^{2}(p-1)(2 p+1) / 2=\left(h-O\left(p^{2}\right)\right) p^{2} .
$$

By Corollary $1, p^{2}=(1-o(1)) \sqrt{l}$. Hence the result.

The following theorem is an immediate consequence of Theorem 1

Theorem 2. Let $G_{1}, G_{2}$ be any two graphs with $\eta\left(G_{1}\right)=h, \eta\left(G_{2}\right)=l$ and $\eta\left(G_{1}\right) \geq$ $\eta\left(G_{2}\right)$. Then $\eta\left(G_{1} \square G_{2}\right)=\eta\left(G_{2} \square G_{1}\right) \geq h \sqrt{l}(1-o(1))$.

\subsection{Tightness of the Lower Bound and the Nonexistence of an Upper Bound}

Let $K_{n}$ and $K_{m}$ be the complete graphs on $n$ and $m$ vertices respectively $(n \geq m)$ and let $h$ be the maximum number such that $K_{h} \preceq K_{n} \square K_{m}$. Let the sets $V_{0}, \ldots, V_{h-1} \subseteq$ $V\left(K_{n} \square K_{m}\right)$ be the pre-images of vertices of $K_{h}$ in $K_{n} \square K_{m}$. Thus, the vertex sets $V_{0}, \ldots, V_{h-1}$ are pairwise disjoint and pairwise adjacent. Moreover, $V_{i}(0 \leq i \leq$ $h-1$ ) induces a connected subgraph in $K_{n} \square K_{m}$.

Without loss of generality, let $n_{0}=\left|V_{0}\right|=\min _{0 \leq i \leq h-1}\left|V_{i}\right|$. Thus $n_{0} \leq \frac{n m}{h}$. For $S \subseteq V\left(K_{m} \square K_{n}\right)$, let $N(S)=\bigcup_{u \in S} N(u)-S$. (Here $N(u)$ denotes the set of the neighbors of $u$ in $K_{n} \square K_{m}$.) Since $K_{h}$ is a complete graph minor of $K_{m} \square K_{n}$, we have:

$$
\left|N\left(V_{0}\right)\right| \geq h-1
$$

Since $V_{0}$ induces a connected graph in $K_{n} \square K_{m}$, the vertices of $V_{0}$ can be ordered $v_{1}, \ldots, v_{n_{0}}$ so that for $2 \leq j \leq n_{0}, v_{j}$ is adjacent to at least one of the vertices in $\left\{v_{1}, \ldots, v_{j-1}\right\}$. Let us define a sequence of sets $\emptyset=X_{0}, X_{1}, \ldots, X_{n_{0}}=V_{0}$ by setting $X_{j}=X_{j-1} \cup\left\{v_{j}\right\}$, for $1 \leq j \leq n_{0}$. Clearly, $\left|N\left(X_{1}\right)\right|=n+m-2$. We claim that 
$\left|N\left(X_{j}\right)\right| \leq\left|N\left(X_{j-1}\right)\right|+n-2$, for $2 \leq j \leq n_{0}$. To see this, recall that $v_{j}$ is adjacent to at least one vertex $v_{k} \in X_{j-1}$. Clearly, out of the $n+m-2$ neighbors of $v_{j}$, at least $m-2$ are neighbors of $v_{k}$ also, and thus are already in $N\left(X_{j-1}\right)$. Now, accounting for $v_{j}$ and $v_{k}$ we have $\left|N\left(X_{j}\right)\right| \leq\left|N\left(X_{j-1}\right)\right|+n-2$, as required. Thus we get $\left|N\left(V_{0}\right)\right|=\left|N\left(X_{n_{0}}\right)\right| \leq n+m-2+\left(n_{0}-1\right)(n-2) \leq n+m-2+\left(\frac{n m}{2}-1\right)(n-2)$. Combining this with Inequality (1) we get:

$$
n+m-2+\left(\frac{n m}{h}-1\right)(n-2) \geq h-1
$$

It is easy to verify that for $h>n \sqrt{m}+m$, the above inequality will not be satisfied. So we infer that $h \leq n \sqrt{m}+m$. (Recalling $n \geq m$, the upper bound tends to $n \sqrt{m}$ asymptotically.)

After proving our lower bound it is natural to ask the following question. Let $G_{1}$ and $G_{2}$ be two arbitrary graphs with $\eta\left(G_{1}\right)=k_{1}$ and $\eta\left(G_{2}\right)=k_{2}$. Does there exists a function $f: N \times N \rightarrow N$ such that $\eta\left(G_{1} \square G_{2}\right) \leq f\left(k_{1}, k_{2}\right)$ ? As examples below show, in general such a function cannot exist.

Let $R_{n}=P_{n} \square P_{n}$ be the $n \times n$ grid graph and let $D_{n}=R_{n} \square K_{2}$ be the $n \times n$ double-grid graph. Since $R_{n}$ is a planar graph, we have $\eta\left(R_{n}\right) \leq 4$. Thus, $D_{n}$ is the Cartesian product of graphs with Hadwiger numbers two and at most four. But, as it was proved in [2], the Hadwiger number of $D_{n}$ is at least $n$. (A sketch of their proof is as follows. Let $G_{1}$ and $G_{2}$ be the two grids $R_{n}$ comprising $D_{n}=R_{n} \square K_{2}$. Observe that there is an edge between any "row" of $G_{1}$ and any "column" of $G_{2}$. Contracting all the rows of $G_{1}$ and all the columns of $G_{2}$ we get a complete bipartite graph $K_{n, n}$ whose Hadwiger number is at least $n+1$.)

Another (even more impressive) example immediately follows from a result of Ivančo, who proved in [8] that $\eta\left(K_{1, n} \square K_{1, n}\right)=2+n$.

\subsection{Consequences of Theorem 2: Hadwiger's Conjecture for Graph Products}

2.3.1. In Terms of Chromatic Number. Theorem 2 naturally leads to the following question: Let $G_{1}$ and $G_{2}$ be any two graphs with $\chi\left(G_{1}\right)=k_{1}$ and $\chi\left(G_{2}\right)=k_{2}$, where $k_{1} \geq k_{2}$. Let $f\left(k_{1}\right)$ be the smallest $m$ such that if $k_{2} \geq m$, then Hadwiger's conjecture holds for $G_{1} \square G_{2}$. Hadwiger's conjecture states that $f\left(k_{1}\right)=1$. Since Hadwiger's conjecture in the most general case seems to be hard to prove, it is interesting to obtain reasonable bounds on $f\left(k_{1}\right)$. We need the following result, proved independently by Kostochka [10] and Thomason [18].

Lemma 5. There exists a constant $c_{2}$ such that for each graph $G, \eta(G) \geq \frac{c_{2} \chi(G)}{\sqrt{\log \chi(G)}}$.

Theorem 2 yields the following result.

Theorem 3. Let $G_{1}$ and $G_{2}$ be any two graphs. There exists a constant $c^{\prime}$ such that if $\chi\left(G_{1}\right) \geq \chi\left(G_{2}\right) \geq c^{\prime} \log ^{1.5}\left(\chi\left(G_{1}\right)\right)$, then Hadwiger's conjecture is true for $G_{1} \square G_{2}$. 
Proof. Let $k_{1}=\chi\left(G_{1}\right)$ and $k_{2}=\chi\left(G_{2}\right)$. Applying Lemma 5 and Theorem 2 and noting that $\sqrt{\sqrt{\log \left(k_{2}\right)}} \leq\left(\sqrt{\log \left(k_{1}\right)}\right)^{0.5}$, we have

$$
\eta\left(G_{1} \square G_{2}\right) \geq c_{1} c_{2}{ }^{1.5} \frac{k_{1} \sqrt{k_{2}}}{\left(\sqrt{\log k_{1}}\right)^{1.5}} .
$$

Now taking $c^{\prime}=\frac{1}{\left(c_{1} c_{2}^{1.5}\right)^{2}},\left(c_{1}\right.$ and $c_{2}$ are the constants that correspond to Theorem $2^{1}$ and Lemma 5 respectively) and recalling that $k_{2} \geq c^{\prime} \log ^{1.5}\left(k_{1}\right)$, we get $\eta\left(G_{1} \square G_{2}\right) \geq k_{1}=\chi\left(G_{1} \square G_{2}\right)$. The latter equality follows from Lemma 1 .

2.3.2. In Terms of Product Dimension. Recall that the product dimension of a connected graph $G$ is the number of prime factors in its (unique) prime factorization. It was shown in [2] that if the product dimension of $G$ is at least $2 \log \chi(G)+3$, then Hadwiger's conjecture is satisfied for $G$. Using theorem 2, we can bring this bound to $2 \log \log \chi(G)+c^{\prime}$, where $c^{\prime}$ is a constant. The following Lemma proved in [2] gives a lower bound for the Hadwiger number of the $d$-dimensional Hypercube, $Q_{d}$.

Lemma 6. $\eta\left(Q_{k}\right) \geq 2^{\lfloor(k-1) / 2\rfloor} \geq 2^{(k-2) / 2}$

Theorem 4. There exists a constant $c^{\prime}$, such that for every connected graph $G$ with product dimension $k$, if $k \geq 2 \log \log \chi(G)+c^{\prime}$, then Hadwiger's conjecture holds for G.

Proof. Let $c^{\prime}=4 \log \frac{1}{c_{1} c_{2}}+3$, where $c_{1}$ and $c_{2}$ are the constants ${ }^{2}$ by Theorem 2 and Lemma 5 respectively. We may assume that $\chi\left(G_{1}\right) \geq \chi\left(G_{i}\right)$, for all $i>1$. By Lemma $1, \chi(G)=\max \left\{\chi\left(G_{1}\right), \chi\left(G_{2}\right), \ldots, \chi\left(G_{k}\right)\right\}=\chi\left(G_{1}\right)$.

Let $X=G_{2} \square G_{3} \square \ldots \square G_{k}$. Since $G$ is connected, each $G_{i}$ is also connected. Moreover, $G_{i}$ has at least two vertices (and hence at least one edge) since $G_{i}$ is prime. It follows that the $(k-1)$-dimensional hypercube is a minor of $X$. Thus by Lemma $6, \eta(X) \geq \eta\left(Q_{k-1}\right) \geq 2^{(k-3) / 2} \geq 2^{\log \log \chi(G)+2 \log \frac{1}{c_{1} c_{2}}}$.

Applying Theorem 2 to $G_{1} \square X$, we get

$$
\eta(G)=\eta\left(G_{1} \square X\right) \geq c_{1} \eta\left(G_{1}\right) \sqrt{\eta(X)}
$$

Recalling that (by Lemma 5), $\eta\left(G_{1}\right) \geq \frac{c_{2} \chi\left(G_{1}\right)}{\sqrt{\log \chi\left(G_{1}\right)}}$, we get $\eta(G) \geq \chi(G)$.

\section{Hadwiger's Conjecture for $\mathbf{G}_{1} \square \mathbf{G}_{2}$ when $\chi\left(\mathbf{G}_{1}\right)=\chi\left(\mathbf{G}_{2}\right)$}

Theorem 3 implies that $G_{1} \square G_{2}$ satisfies Hadwiger's conjecture if $\chi\left(G_{1}\right)=\chi\left(G_{2}\right)=$ $t$ is sufficiently large. ( $t$ has to be sufficiently large, because of the constant $c^{\prime}$ involved

\footnotetext{
1 By Theorem 2 we have $\eta\left(G_{1} \square G_{2}\right) \geq c_{1} h \sqrt{l}$, where $c_{1}$ is a constant.

$2 c_{1}, c_{2} \leq 1$. So $\frac{1}{c_{1} c_{2}} \geq 1$
} 
in Theorem 3). In this section we give a different proof for this special case. We show that irrespective of the value of $t\left(=\chi\left(G_{1}\right)\right), G_{1} \square G_{2}$ satisfies Hadwiger's conjecture if $\chi\left(G_{1}\right)=\chi\left(G_{2}\right)$.

A graph $G$ is said to be $k$-critical if $\chi(H)<\chi(G)=k$ for every proper subgraph $H$ of $G$. Every $k$-chromatic graph contains a $k$-critical subgraph.

Theorem 5. If $\chi(G)=\chi(H)$, then Hadwiger's conjecture is true for $G \square H$.

Proof. Let $\chi(G)=\chi(H)=n$. Let $G^{\prime}$ and $H^{\prime}$ be $n$-critical subgraphs of $G$ and $H$ respectively. Then $\delta\left(G^{\prime}\right) \geq n-1$ (see [21]) and hence $K_{1, n-1} \preceq G^{\prime}$. Similarly $K_{1, n-1} \preceq H^{\prime}$. Thus we have $\eta(G \square H) \geq \eta\left(G^{\prime} \square H^{\prime}\right) \geq \eta\left(K_{1, n-1} \square K_{1, n-1}\right)$. By Ivančo's [8] result, we have $\eta\left(K_{1, n-1} \square K_{1, n-1}\right)=2+(n-1)>n$. This together with Lemma 1, gives $\eta(G \square H)>n=\chi(G \square H)$, proving the Theorem.

It was shown in [2] that if a graph $G$ is isomorphic to $F^{d}$, for some graph $F$ and $d \geq 3$ then Hadwiger's conjecture is true for $G$. The following improvement is an immediate consequence of Theorem 5 and Lemma 1.

Theorem 6. Let a graph $G$ be isomorphic to $F^{d}$ for some graph $F$ and for $d \geq 2$. Then Hadwiger's conjecture is true for $G$.

Acknowledgements. We thank the referees for helpful comments.

\section{References}

1. Aurenhammer, F., Hagauer, J., Imrich, W.: Cartesian graph factorization at logarithmic cost per edge. Computational Complexity 2, 331-349 (1992)

2. Chandran, L.S., Sivadasan, N.: On the Hardwiger's conjecture for graph products. Discrete Mathematics 307, 266-273 (2007)

3. Diestel, R.: Graph Theory. Springer Verlag, New York (2000)

4. Diestel, R., Rempel, C.: Dense minors in graphs of large girth. Combinatorica 25, 111$116(2005)$

5. Dirac, G.A.: In abstrakten Graphen vorhandene vollständige 4-Graphen und ihre Unterteilungen. Math. Nachr. 22, 61-85 (1960)

6. Hadwiger, H.: Über eine Klassifikation der Streckenkomplexe, Vierteljscr. Naturforsch. Gessellsch. Zürich 88, 132-142 (1943)

7. Imrich, W., Klavžar, S.: Product Graphs: Structure and Recognition. Wiley, New York (2000)

8. Ivančo, J.: Some results on the Hadwiger number of graphs. Math. Slovaca 38, 221-226 (1988)

9. Iwaniec, H., Pintz, J.: Primes in short intervals. Monatsh. Math. 98, 115-143 (1984)

10. Kostochka, A.V.: The minimum hadwiger number of graphs with a given mean degree of vertices, Metody Diskret. Analiz. 38, 37-58 (1982) (In Russian)

11. Kotlov, A.: Minors and strong products. European Journal of Combinatorics 22, 511$512(2001)$

12. Kühn, D., Osthus, D.: Minors in graphs of large girth. Random Structures and Algorithms 22, 213-225 (2003)

13. Mader, W.: Homomorphiesätze für Graphen. Math. Annalen 178, 154-168 (1968) 
14. Miller, Z.: Contractions of graphs: A theorem of Ore and an extremal problem. Discrete Mathematics 21, 261-273 (1978)

15. Robertson, N., Seymour, P.D., Thomas, R.: Hadwiger's conjecture for K6-free graphs. Combinatorica 13, 279-361 (1993)

16. Ryser, H.J.: Combinatorial mathematics, The Carus Mathematical Monographs. 14 Wiley, New York (1963)

17. Sabidussi, G.: Graphs with given group and given graph theoretic properties. Canad. J. Math. 9, 515-525 (1957)

18. Thomason, A.G.: An extremal function for contractions of graphs. Math. Proc. Camb. Phil. Soc. 95, 261-265 (1984)

19. Wagner, K.: Über eine Eigenschaft der Ebenen Komplexe. Math. Annalen 114, 570-590 (1937)

20. Wagner, K.: Beweis einer Abschwächung der Hadwiger-Vermutung. Math. Annalen 153, 139-141 (1964)

21. West, D.B.: Introduction to Graph Theory. Prentice Hall India, NewDelhi (2003)

Received: July 17, 2007

Final Version received: March 20, 2008 